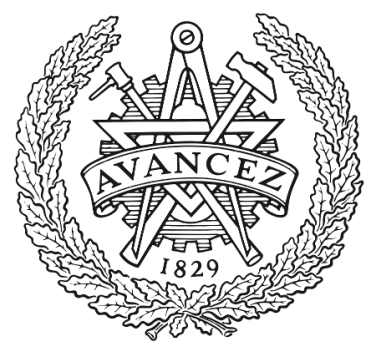

CHALMERS

UNIVERSITY OF TECHNOLOGY

\title{
Experimental Demonstration of 8-Dimensional Voronoi Constellations with 65,536 and $16,777,216$ Symbols
}

Downloaded from: https://research.chalmers.se, 2023-04-26 06:43 UTC

Citation for the original published paper (version of record):

Mirani, A., Vijayan, K., He, Z. et al (2021). Experimental Demonstration of 8-Dimensional Voronoi Constellations with 65,536 and 16,777,216

Symbols. 2021 European Conference on Optical Communication, ECOC 2021.

http://dx.doi.org/10.1109/ECOC52684.2021.9605988

N.B. When citing this work, cite the original published paper. 


\title{
Experimental Demonstration of 8-Dimensional Voronoi Constellations with 65,536 and 16,777,216 Symbols
}

\author{
Ali Mirani ${ }^{(1)}$, Kovendhan Vijayan ${ }^{(1)}$, Zonglong He ${ }^{(1)}$, Shen $\mathrm{Li}^{(2)}$ \\ Erik Agrell(2), Jochen Schröder ${ }^{(1)}$, Peter Andrekson ${ }^{(1)}$, Magnus Karlsson ${ }^{(1)}$
}

(1) Photonics Laboratory, Department of Microtechnology and Nanoscience, Chalmers University of Technology, Gothenburg, Sweden, mirani@chalmers.se

(2) Department of Electrical Engineering, Chalmers University of Technology, Gothenburg, Sweden

\begin{abstract}
We experimentally demonstrate high-cardinality, low-complexity Voronoi constellations based on the E8 lattice over multiple time slots with OSNR and launch power gains of up to 1.7 and $2.4 \mathrm{~dB}$ for back-to-back and $80 \mathrm{~km}$ fiber transmission, respectively, compared to QAM formats.
\end{abstract}

\section{Introduction}

To meet the ever-growing demands of network traffic, decreasing the asymptotic $1.53 \mathrm{~dB}$ gap to the Shannon limit and increasing the spectral efficiency (SE) are essential. Signal shaping can close the shaping gap, classified into probabilistic shaping (PS) and geometric shaping (GS). On the other hand, the SE can be increased by utilizing multidimensional constellations due to the improvement in the asymptotic power efficiency (APE), i.e., improved sensitivity with respect to quadrature phaseshift keying (QPSK) 1 .

Developments in coherent optical detection have enabled exploiting the full-dimensional capacity of optical communication systems by utilizing the available dimensions of light, i.e., amplitude, phase, time, wavelength, polarization and space. By joint optimization, power-efficient multidimensional modulation formats were first studied for optical communications in [2]-4]. In [5], a 4-dimensional (4D) lattice-based modulation format was experimentally demonstrated and compared with polarizationmultiplexed 16 quadrature amplitude modulation (QAM). Using 8 dimensions, higher sensitivity and transmission reach were demonstrated and compared to QAM formats employing amplitude, phase, polarization, wavelengths $\left[\right.$, and time slots $\frac{7]}{7]}$. Experimental demonstrations were performed using multicore fibers transmitting 12-[9] and 16dimensional[10] modulation formats. Up to $24 \mathrm{di}-$ mensions were studied in simulations over the nonlinear fiber channel[11], [12]. However, improving the SE using multidimensional constellations increases the computational complexity of generation and detection as it involves full enumeration of constellation points with look-up tables.

In this work, the performance of low-complexity

978-1-6654-3868-1/21\$31.00 @2021 IEEE
Voronoi constellations is studied for the first time, to the best of our knowledge, experimentally in 8 dimensions by performing noise loading and fiber transmission with two different symbol rates (10 and 20 GBaud). We implement the 8-dimensional (8D) Gosset lattice (E8) using amplitude and phase (in-phase and quadrature (I/Q)) components as well as 4 time slots. Two constellations, E8B2 and E8B3, are considered with sizes of $2^{16}$ and $2^{24}$, respectively. The constellations are generated and detected algorithmically without any look-up tables. We show that, at a SE of $4 \mathrm{bits} / \mathrm{sym} / \mathrm{pol}$, a bit error rate (BER) of $10^{-3}$, and 10 GBaud symbol rate, the optical signal-to-noise ratio (OSNR) and launch power gains are $0.7 \mathrm{~dB}$ with respect to 16QAM, and at 20 GBaud, these gains are 0.6 and $0.4 \mathrm{~dB}$, respectively. Moreover, at 6 bits $/ \mathrm{sym} / \mathrm{pol}$ with 10 GBaud symbol rate and a BER of $10^{-3}$, we demonstrate OSNR and launch power gains of 1.7 and $2.4 \mathrm{~dB}$ compared to 64QAM.

\section{Voronoi Constellations}

A Voronoi constellation is a set of vectors in the multidimensional space based on two lattices: a shaping lattice and a coding lattice $\frac{133}{1,14}$. The outer boundary of the constellation is determined by the scaled Voronoi region of the shaping lattice and the constellation symbols are selected from the coding lattice vectors inside this boundary ${ }^{[15}$. For instance, QAM formats are generated using integer (cubic) lattices for both the shaping and coding lattices, providing $0 \mathrm{~dB}$ shaping and coding gains ${ }^{[14]}$. Usually, QAM formats are used as a benchmark to compare the performance of other modulation schemes ${ }^{[9]},[10]$. On the other hand, the E8 lattice provides the densest packing of $8 \mathrm{D}$ hyperspheres (coding gain) and the Voronoi region of the $\mathrm{E} 8$ lattice has a $0.65 \mathrm{~dB}$ shaping gain compared to an $8 \mathrm{D}$ hypercube boundary. The E8 constellation with the 


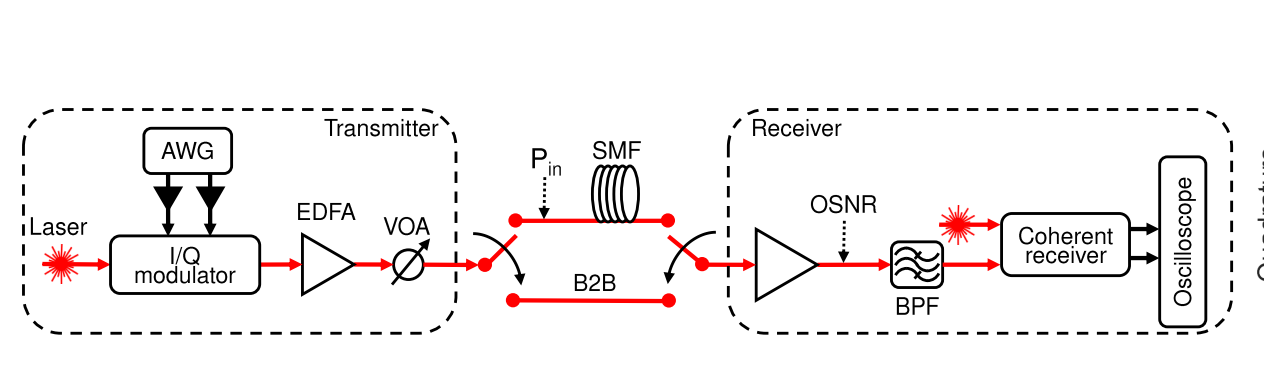

(a)

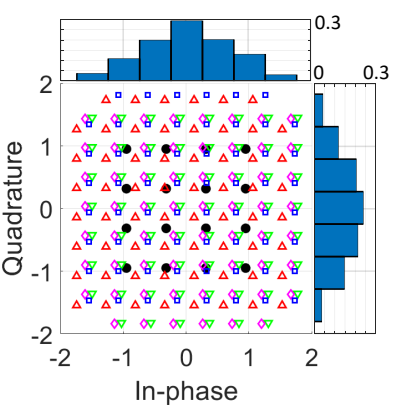

(b)

Fig. 1: (a) Block diagram of the experimental setup for the optical power sweep in the single span fiber transmission and the back-to-back OSNR sweep. (b) 2D representation of E8B2 constellation at 4 bits/sym/pol with 4 time slots at unit average energy. Different markers show the symbols in different time slots. Black solid circles indicate the QAM format at the same spectral efficiency. The blue bars indicate the normalized histogram of the E8B2 constellation points along the real and imaginary axes.

SE of 4 bits/sym/pol is named as E8B2 (E8 with 2 bits per dimension) consisting of 65536 symbols. In the case of E8 with 6 bits/sym/pol, 16777216 symbols are obtained, known as E8B3 (E8 with 3 bits per dimension). For these constellations, fast and low-complexity algorithms for bit-to-symbol mapping, detection algorithm (closest point algorithm), and symbol-to-bit demapping were studied in 121,15 .

To transmit multidimensional modulation formats in a real system, each constellation dimension needs to be realized by a physical dimension. In this work, we use 4 consecutive time slots, $I / Q$ components in each time slots, one polarization, and one carrier wavelength. The realization of constellation symbols in 2 dimensions are shown in Fig. 1(b) for the E8B2 constellation. The dimensions of $8 \mathrm{D}$ symbols are combined to create $\mathrm{I} / \mathrm{Q}$ components and they are rotated 45 degrees compared with E8 lattice in $\frac{13]}{10}$ to generate fewer levels along each axes. The normalized histograms show the probability of 2-dimensional (2D) symbols along each axes. It can be seen that the uniform multidimensional GS constellation results in a PS constellation when projected onto lower dimensions.

\section{Experimental Setup}

We compared the Voronoi constellations against QAM formats of the same SE by noise loading and fiber transmission experiments. The setup is shown in Fig. 1(a). The optical carrier generated from an external cavity laser (ECL) of linewidth $\leq 100 \mathrm{kHz}$ operating at $1550.1 \mathrm{~nm}$ was modulated by an $\mathrm{I} / \mathrm{Q}$ modulator driven by the amplified electrical signals from an arbitrary waveform generator (AWG), with $2^{15}$ (10 Gbaud) and $2^{16}$ symbol $(20$ Gbaud) frames. For both symbol rates, the frames consisted of pilot and payload symbols shaped with root-raised cosine filters of $10 \%$ roll-off. The pilots were QPSK symbols adding 6.15 and $4.64 \%$ overhead at 10 and 20 GBaud, respectively, and assisted in the digital signal processing (DSP) at the receiver [16],[17]. For Voronoi constellations, the payloads were the E8 constellation symbols over 4 consecutive time slots. The I/Q modulator nonlinearity compensation, digital-to-analog converter frequency response compensation, and clipping were performed at the transmitter to improve the optical back-to-back (B2B) performance. The modulated signal was then amplified with a booster erbium-doped fiber amplifier (EDFA). A variable optical attenuator (VOA) was used to sweep the power into the pre-amplifier changing the OSNR for noise loading and to set the required launch power, $\mathrm{P}_{\text {in }}$, at the transmission span input in case of the fiber transmission. An $80 \mathrm{~km}$ single mode fiber (SMF) was used as the transmission span with loss parameter $\alpha=0.2 \mathrm{~dB} / \mathrm{km}$, dispersion parameter $D=16.8 \mathrm{ps} / \mathrm{nm} / \mathrm{km}$, and nonlinear parameter $\gamma=1.3\left(\mathrm{~W} \mathrm{~km}^{-1}\right.$.

After the pre-amplifier, the signal was filtered to remove the out-of-band noise and fed to the coherent receiver. A free-running $\mathrm{ECL}$ with $\leq 100 \mathrm{kHz}$ linewidth at $1550.1 \mathrm{~nm}$ was used as a local oscillator. The electrical signals from the coherent receiver were measured using a real-time oscilloscope and were processed offline using ${ }^{[17}$ to compensate the channel and receiver impairments. For the QAM formats, Gray labeling and maximum likelihood detection were used. However, for Voronoi constellations, quasi-Gray labeling and the closest point algorithm were applied to recover the transmitted bits as in 122 .

The signal and the noise power were measured using an optical spectrum analyzer with a resolution bandwidth of $0.5 \mathrm{~nm}$. The OSNR was calculated by integrating the noise power in a bandwidth of $0.1 \mathrm{~nm}$. The input power to the span, $P_{\text {in }}$, was measured with a power-calibrated OSA using a resolution bandwidth of $0.5 \mathrm{~nm}$.

\section{Results and Discussions}

The uncoded BER performance of the E8 lattice is compared with QAM formats with the same SE. In 


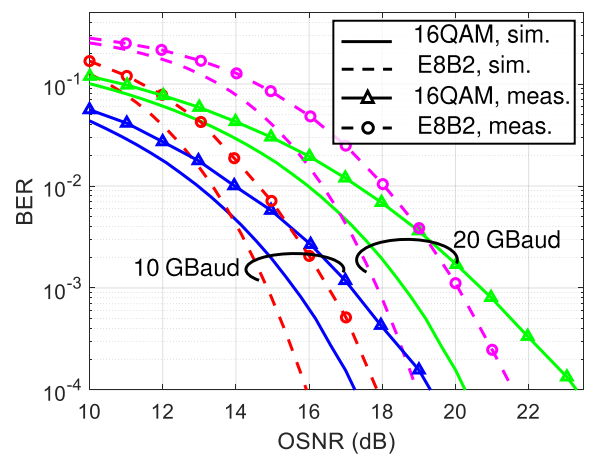

(a)

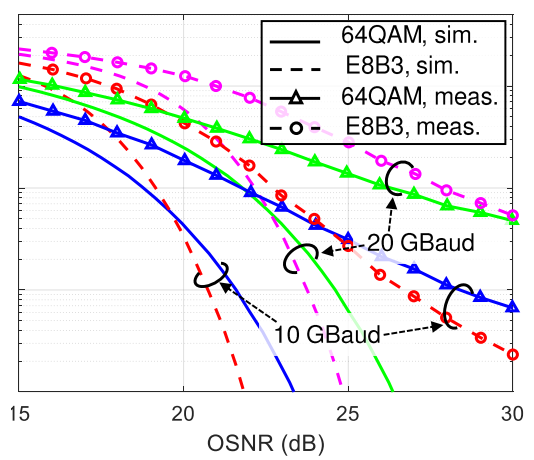

(b)

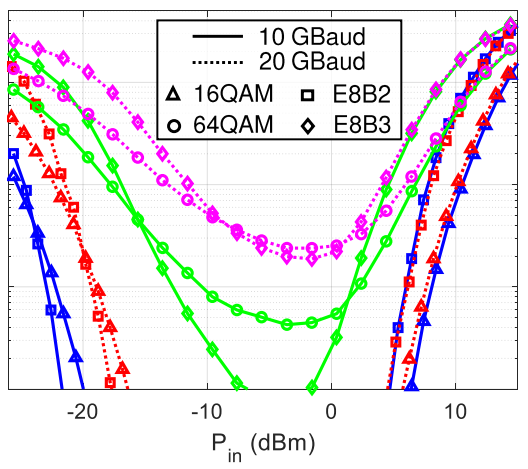

(c)

Fig. 2: (a) Measured and simulated BER vs. OSNR at an SE of 4 bits/sym/pol. Monte Carlo simulations are based on additive white Gaussian noise as the only impairment. Lines with markers indicate experimental measurements and lines without markers indicate simulations. (b) Measured and simulated BER vs. OSNR at an SE of 6 bits/sym/pol. (c) Measured BER vs. launch power at the input of the $80 \mathrm{~km}$ fiber span.

Fig. 2(a), the E8B2 Voronoi constellation is compared with 16QAM with SE $=4$ bits/sym/pol at symbol rates of 10 and 20 GBaud. The implementation penalty is calculated as the difference between OSNRs of the simulated and measured values. The implementation penalty at BER $=10^{-2}$ is 1 and $1.35 \mathrm{~dB}$ for 10 and 20 GBaud 16QAM, respectively, and for E8B2 is 1.25 and $1.6 \mathrm{~dB}$. Below a BER of approximately $5.2 \times 10^{-3}$ and $3.5 \times 10^{-3}$ for simulations and experiments, respectively, E8B2 outperforms 16QAM because of higher APE. However, at higher BERs, 16QAM outperforms E8B2 because of Gray labeling. At BER $=10^{-3}$, E8B2 requires 0.7 and $0.6 \mathrm{~dB}$ less OSNR compared to 16QAM at 10 and 20 GBaud, respectively.

In Fig. 2(b), a similar comparison is performed between E8B3 and 64QAM at SE $=6$ bits/sym/pol. The implementation penalty increases for the higher-order modulation formats due to the reduced effective number of bits in the digital-toanalog and analog-to-digital converters at high symbol rates and the bandwidth limitations of transceiver components. The BER crossing point for simulations and experiments are approximately $5.8 \times 10^{-3}$ and $3.7 \times 10^{-3}$, respectively. At very high OSNRs, there is an error floor for E8B3 and 64QAM due to the limited electrical signal-to-noise ratio in the transmitter, where E8B3 outperforms 64QAM. At $\mathrm{BER}=10^{-3}$, E8B3 requires $1.7 \mathrm{~dB}$ less OSNR compared to 64QAM at 10 GBaud.

We also compare the performance of the E8 and QAM in fiber transmission. In Fig. 2(c), the BER is plotted for different launch powers with SEs of 4 and 6 bits/sym/pol at symbol rates of 10 and 20 GBaud. In the linear regime, similar to Fig. 2(a) and 2(b), E8B2 and E8B3 outperform 16QAM and 64QAM at BER $<4 \times 10^{-3}$, respectively. The launch power gains at $B E R=10^{-3}$ is approximately $0.7,0.4$, and $2.4 \mathrm{~dB}$ for $\mathrm{E} 8 \mathrm{~B} 2$ at 10 and
20 GBaud and E8B3 at 10 GBaud, respectively, compared to 16QAM and 64QAM at the same symbol rate. By increasing the launch power to the fiber, nonlinearity increases and there is an optimum launch power with minimum BER because of the balance between the noise and the nonlinearity distortion. The optimum points are not shown for E8B2 and 16QAM since they happen at $\mathrm{BER}<4 \times 10^{-3}$; however, in all cases, E8 constellations outperform QAM at the optimum launch power. By further increasing the launch power after the optimum point, the QAM formats start to outperform E8 constellations in the nonlinear regime.

Therefore, due to the improved performance at BER $<4 \times 10^{-3}$, E8 constellations can be combined with hard-decision forward error correction (FEC) codes, e.g., KP4 [18], to be used in lowlatency and low-complexity applications. At higher BERs, the QAM formats outperform E8 and they can be used with higher pre-FEC limits.

\section{Conclusions}

We have experimentally demonstrated Voronoi constellations based on the E8 lattice for the first time in optical communication systems. With low-complexity modulation and demodulation algorithms, constellations with $2^{16}$ and $2^{24}$ symbols are transmitted and detected. At BERs approximately below $4 \times 10^{-3}$, the OSNR and launch power gains are shown with respect to QAM formats at the same SE in the back-to-back and fiber transmission. Therefore, combined with hard-decision FECs at low pre-FEC limits, these Voronoi constellations can be suitable for low-latency and lowcomplexity applications.

\section{Acknowledgements}

We wish to acknowledge financial support from the Knut and Alice Wallenberg foundation as well as the Swedish Research Council (VR) in projects 2015-00535, 2017-03702 and 2019-04078. 


\section{References}

[1] M. Karlsson and E. Agrell, "Power-efficient modulation schemes", in Impact of Nonlinearities on Fiber Optic Communications, Springer, 2011, pp. 219-252.

[2] H. Bülow, "Polarization QAM modulation (POL-QAM) for coherent detection schemes", in Optical Fiber Communications Conference (OFC), 2009.

[3] M. Karlsson and E. Agrell, "Which is the most powerefficient modulation format in optical links?", Optics Express, vol. 17, no. 13, pp. 10814-10819, 2009.

[4] E. Agrell and M. Karlsson, "Power-efficient modulation formats in coherent transmission systems", Journal of Lightwave Technology, vol. 27, no. 22, pp. 5115-5126, 2009.

[5] T. A. Eriksson, S. Alreesh, C. Schmidt-Langhorst, F. Frey, P. W. Berenguer, C. Schubert, J. K. Fischer, P. A. Andrekson, M. Karlsson, and E. Agrell, "Experimental investigation of a four-dimensional 256-ary lattice-based modulation format", in Optical Fiber Communications Conference (OFC), 2015

[6] T. A. Eriksson, P. Johannisson, M. Sjödin, E. Agrell, P. A. Andrekson, and M. Karlsson, "Frequency and polarization switched QPSK", in European Conference on Optical Communication (ECOC), 2013.

[7] M. Nakamura, F. Hamaoka, A. Matsushita, K. Horikoshi, H. Yamazaki, M. Nagatani, A. Sano, A. Hirano, and Y. Miyamoto, "Coded eight-dimensional QAM technique using iterative soft-output decoding and its demonstration in high baud-rate transmission", Journal of Lightwave Technology, vol. 35, no. 8, pp. 1369-1375, 2017.

[8] B. Chen, C. Okonkwo, H. Hafermann, and A. Alvarado, "Eight-dimensional polarization-ring-switching modulation formats", IEEE Photonics Technology Letters, vol. 31, no. 21, pp. 1717-1720, 2019.

[9] R.-J. Essiambre, R. Ryf, S. van der Heide, J. I. Bonetti, H. Huang, M. Kodialam, F. J. García-Gómez, E. C. Burrows, J. C. Alvarado-Zacarias, R. Amezcua-Correa, et al., "First Transmission of a 12D Format Across Three Coupled Spatial Modes of a 3-Core Coupled-Core Fiber at 4 bits/s/Hz", in Optical Fiber Communications Conference (OFC), 2020.

[10] G. Rademacher, B. Puttnam, R. Luis, Y. Awaji, N. Wada, E. Agrell, and K. Petermann, "Experimental investigation of a 16-dimensional modulation format for long-haul multicore fiber transmission", in European Conference on Optical Communication (ECOC), 2015.

[11] D. S. Millar, T. Koike-Akino, S. Ö. Arık, K. Kojima, K. Parsons, T. Yoshida, and T. Sugihara, "High-dimensional modulation for coherent optical communications systems", Optics Express, vol. 22, no. 7, pp. 8798-8812, 2014.

[12] A. Mirani, E. Agrell, and M. Karlsson, "Low-complexity geometric shaping", Journal of Lightwave Technology, vol. 39, no. 2, pp. 363-371, 2020.

[13] J. H. Conway and N. J. A. Sloane, Sphere Packings, Lattices and Groups, 3rd ed. Springer, 1999.

[14] G. D. Forney, Jr., "Multidimensional Constellations-Part II: Voronoi Constellations", IEEE Journal on Selected Areas in Communications, vol. 7, no. 6, pp. 941-958, 1989.

[15] J. Conway and N. Sloane, "A fast encoding method for lattice codes and quantizers", IEEE Transactions on Information Theory, vol. 29, no. 6, pp. 820-824, 1983.
[16] M. Mazur, J. Schröder, A. Lorences-Riesgo, T. Yoshida, M. Karlsson, and P. A. Andrekson, "Overheadoptimization of pilot-based digital signal processing for flexible high spectral efficiency transmission", Optics express, vol. 27, no. 17, pp. 24654-24669, 2019.

[17] J. Schröder and M. Mazur, QAMPy a DSP chain for optical communications, 2018. DOI: 10 . 5281 / zenodo . 1195720

[18] E. Agrell and M. Secondini, "Information-theoretic tools for optical communications engineers", in IEEE Photonics Conference (IPC), 2018. 